Association for Information Systems AIS Electronic Library (AISeL)

Wirtschaftsinformatik Proceedings 2003

Wirtschaftsinformatik

September 2003

\title{
Business Process Modelling and Enactment for Task-Specific Information Support
}

Giorgos Papavassiliou

National Technical University of Athens

Spyridon Ntioudis

National Technical University of Athens

Gregory Mentzas

National Technical University of Athens

Andreas Abecker

Forschungszentrum Informatik (FZI) Karlsruhe, abecker@fzi.de

Follow this and additional works at: http://aisel.aisnet.org/wi2003

\section{Recommended Citation}

Papavassiliou, Giorgos; Ntioudis, Spyridon; Mentzas, Gregory; and Abecker, Andreas, "Business Process Modelling and Enactment for Task-Specific Information Support" (2003). Wirtschaftsinformatik Proceedings 2003. 51.

http://aisel.aisnet.org/wi2003/51

This material is brought to you by the Wirtschaftsinformatik at AIS Electronic Library (AISeL). It has been accepted for inclusion in Wirtschaftsinformatik Proceedings 2003 by an authorized administrator of AIS Electronic Library (AISeL). For more information, please contact elibrary@aisnet.org. 
In: Uhr, Wolfgang, Esswein, Werner \& Schoop, Eric (Hg.) 2003. Wirtschaftsinformatik 2003: Medien - Märkte - Mobilität, 2 Bde. Heidelberg: Physica-Verlag

ISBN: 3-7908-0111-9 (Band 1)

ISBN: 3-7908-0116-X (Band 2)

(C) Physica-Verlag Heidelberg 2003 


\title{
Business Process Modelling and Enactment for Task-Specific Information Support
}

\author{
Giorgos Papavassiliou, Spyridon Ntioudis, Gregory Mentzas
}

National Technical University of Athens

\author{
Andreas Abecker \\ Forschungszentrum Informatik (FZI) Karlsruhe
}

\begin{abstract}
IT support for knowledge workers in their daily work can take many different guises: groupware systems and information retrieval tools help to exploit and develop function knowledge (how to perform a given activity, or function), while workflow management systems address the process knowledge (which activities to perform in which temporal-logical order, normally modelled in a rigid manner for clearly structured processes). However, what is missing so far in business-process oriented knowledge management, is an environment that (i) integrates the business process and knowledge management aspects of weakly structured knowledge work, and (ii) actively supports the worker in using and adding knowledge resources. This paper presents an approach to support knowledge-intensive business processes. As an example we sketch a case study from the Greek public sector.
\end{abstract}

Keywords:Business-Process Oriented Knowledge Management

\section{Introduction}

One of the most commonly mentioned barriers for Knowledge Management (KM) in organisations is the lack of time. Therefore, KM activities have to be integrated into daily work. On the other hand, most Business Process Management (BPM) efforts have not focused much on knowledge. This is indeed critical, considering that knowledge is treated more and more as a principal success factor - or the major driving force behind business success. Moreover, although business process modelling tools and/or workflow management systems support in an adequate manner the modelling and enactment of business processes, they often lack appropriate support for knowledge-related activities.

From the above, it becomes clear that an approach is missing that explicitly integrates $\mathrm{KM}$ activities into the business process environment. In the present paper 
we attempt to fill that gap by developing and testing methods and tools for proactive, context-sensitive delivery of knowledge in such processes. Our work builds on the artificial intelligence approach to organisational memories [Reimer et al. (2000)] and extends the work of other researchers in the field of integrating organisational memories with workflow management [Staab et al. (2000), Van Kaathoven et al. (1999)]. The main characteristics is the explicit treatment of the knowledge-intensive, weakly-structured character of decision-oriented processes or process parts.

Our approach to weakly-structured workflow aims at combining a project management tool's flexibility with the complex task handling and distribution aspects of traditional workflow systems. It provides open points allowing for later process refinement at runtime and flexible change possibilities facilitating process adaptation on the fly [Klein et al. (2000)]. Our modelling tool extends the formalisms used in most existing business process modelling tools [Yu and Wright (1997)] supporting in an integrated manner the modelling of weakly-structured processes and domain knowledge structures.

We believe that our IT solution must be introduced in a company with appropriate methodological guidance and modelling tools. For this reason, we developed a Business Knowledge Method that integrates modelling and management of processes and knowledge.

Finally, we present the application of our approach to a knowledge-intensive business process of the largest Greek Social Security organisation. We have selected the process of granting full old age pension to insured people, which is, to some extent, a straightforward and well-defined business process. Nevertheless it contains critical steps for finding a decision which are knowledge and document intensive. In the case we examine, the steps of the process are often done under uncertainty, they are influenced by many legal regulations, and they are vital for the correct result of the process.

The paper is structured as follows. The next section outlines the requirements for supporting knowledge-intensive business processes and provides a short overview of our approach. Section 3 illustrates the developed modules of our method, while Section 4 describes the tools for modelling and enacting business processes. Section 5 pinpoints the relationship of our approach to public administration processes and describes its application to the social security business process. Finally, the last section 6 concludes with some related work and discusses some directions for further research. 


\section{Supporting Knowledge-Intensive Processes}

An analysis of knowledge work [Buckingham Shum (1998)] shows that knowledge-intensive processes are characterized by dynamic changes of goals, fluid information environment, unexpected constraints, and highly individual and ad-hoc communication and collaboration patterns. Moreover, knowledge generation and application play an important role. Such processes have to be analysed from a KM perspective, and KM activities should be integrated into daily work. Support of this type of work seems difficult to achieve, but is nevertheless desirable.

Existing systems, like project management and workflow management systems (WfMS), present limitations that restrict their usability in such an environment. Project management tools - although flexible enough to support unique and dynamic processes - fall short in modelling and enacting such processes. In addition, there is no possible support for context-sensitive information using such tools. On the other hand, traditional WfMS facilitate the modelling and execution of complex processes, but they exhibit major limitations when confronting adaptivity issues and support for knowledge related tasks. The reason for this is that current workflow approaches are not flexible enough to adapt on the fly to changing processes. Moreover, the knowledge needed for executing the processes is not explicitly described in the workflow model.

A possible combination of the two types of systems can potentially provide adequate support for knowledge-intensive processes. More specifically, a system that combines the flexibility of a project management tool with the complexity supported by workflow tools should demonstrate the following characteristics:

WfMS as an assistant: the workflow will not follow a prescriptive, but a descriptive philosophy; the system offers to the user tasks and processes which can cooperatively be solved or changed. A continuous process improvement is aimed at. Hence the main system benefit is not so much automatic task delegation, load distribution etc, but more a "planning help", an "advanced project management tool", a documentation instrument, and a collaboration platform.

Hierarchical decomposition of tasks: processes are modelled as hierarchical task decompositions with several possible instantiations for specific subtasks. Subtask definitions maybe found in task ontologies describing the kinds of activities occurring in the organisation.

Modelling and enactment interleaved: starting from the idea of highly individual and ad-hoc work in a knowledge-intensive business, we expect stored task and process models to be usually incomplete or insufficient for a task at hand, to be refined, changed, or extended at runtime.

Expressive process logic: since the temporal / logical interdependencies of tasks and subtasks might be more complex and difficult to articulate than it is possible in simple process definition languages we expect here an expressive language for 
task execution preconditions useful which might refer to the current task context, time conditions, or arbitrary domain or resource specific logical constraint.

Context-sensitive information management: providing contextually selected, task and user specific knowledge is a main goal of the whole approach. In detail, we should be able to couple the WfMS in such a way with other systems, that it is possible to access the function knowledge in an optimal manner. Ideally, the notion of context provided by the workflow system - which "knows" what the user is currently expected to do, what he did before and what he shall do after this task - should be employed in order to optimally incorporate external tools, e.g. by accessing an information retrieval (IR) system already with the correct search request, or starting a collaboration tool already with a connection to a person which is known (from the yellow page system) to be knowledgeable at the topic in quest.

Another starting point is the observation that explicit knowledge relevant for a specific task or a decision is normally spread over many different kinds of documents, forms, legislative texts, etc. Project teams in knowledge-intensive business processes deal with a huge amount of information. Lessons learned in previous engagements, insights from prior projects, notes for subsequent process steps are scattered among manifold "knowledge containers", from the personal memory, over paper, to different electronic systems. Even if there are mechanisms to explicitly capture and store bits and pieces of "best practice", these are seldom used in a coordinated manner, and at best take into account document content, but not so much document context, i.e. neither the creation situation nor the potential usage situation. On the other hand, business processes are a context-giving, structuring element prevalent in a company, often even formally modelled for some purpose such that it would make sense to exploit the usage of business processes to organise knowledge archives. The goal would be to enable context-sensitive storage, more purposeful access to information, and better integration with the processoriented, day-to-day work of the employee with the knowledge system.

Coming from these requirements, some major design decisions of our approach look as follows: We employ formally modelled business processes (BPs) as an ontology which can be used to specify the creation, or the potential usage context, or both, for a given knowledge item. This leads to the idea of a process-oriented structured archive, a meta information system providing conceptual structures to access the underlying legacy systems.

For achieving active knowledge delivery and storage, we employ a WfMS as the host, which is aware of the specific tasks to be performed by the user at a given point in time. Weakly-structured workflow models represent knowledge-intensive work routines which are usually not so strict and predetermined as, e.g., administrative workflows. Enriched workflow models describe information flow in the process and information needs for specific tasks. An information assistant observes the running workflow and interprets modelled information needs to offer active support from the process-oriented structured archive; further it maintains a 
notion of IR context using the modelled information flow variables which allows for more precise queries to the archive. Task context can also be used for information storage to describe the creation context of a given knowledge item.

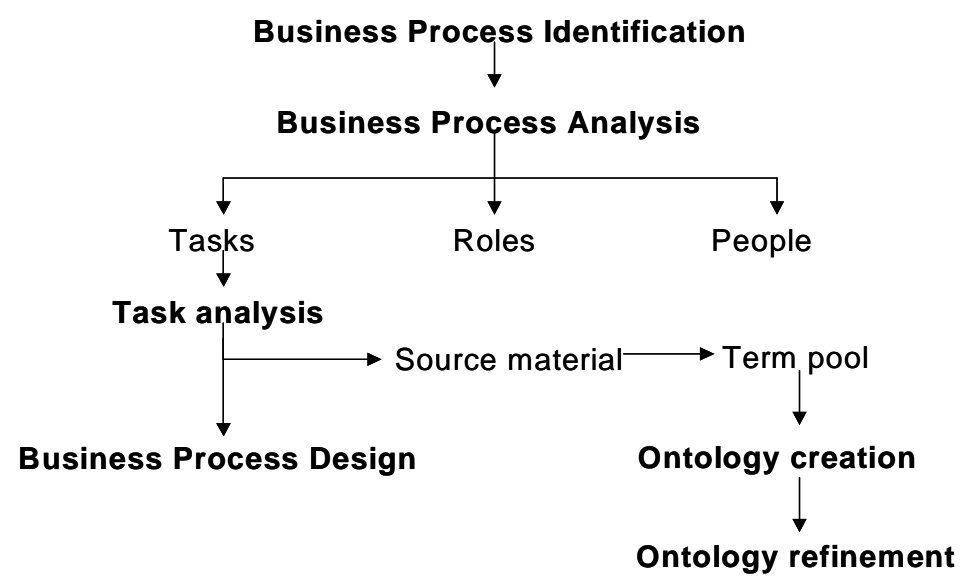

Figure 1: Overview of the Business Knowledge Method

\begin{abstract}
Altogether, existing knowledge sources are used and extended in a more efficient and more consistent way throughout the company. However, the above scenario is based upon several analysis and modelling steps. (1) Business processes and domain ontologies for knowledge organisation / content description, (2) weaklystructured workflows for knowledge-intensive business processes, and (3) information flow and information needs for workflow enrichment, must be acquired and maintained over time. Further, the overall approach must be introduced in a company in the larger context of a comprehensive KM or BPM initiative. All required steps should be supported with appropriate methodological guidance and tools. Consequently, we developed the following method [Papavassiliou et al., 2002].
\end{abstract}

\title{
3 The DECOR Business Process Knowledge Method
}

There is an imperative need for a structured approach for performing business process oriented knowledge projects. This approach ought to support all necessary phases with appropriate methodological guidance and tools. The Business Process Knowledge Method provides the methodological guidance towards that direction amalgamating elements from the CommonKADS [Schreiber et al. (1999)] and the IDEF5 [IDEF5 (2000)] methods. Figure 1 provides an overview of the method.

Step 1: Business Process Identification: This activity involves the identification of the most appropriate business process/-es to be used as the central point for the 
development of the ontology. The selection of the most appropriate business process(es) can be based on the criteria Knowledge intensity and Process complexity taken from [Heisig (2000)].

Step 2: Business Process Analysis: This activity involves a general description of the selected business process/-es in terms of (a) tasks; (b) roles involved; and (c) key people and source material.

Step 3: Task Analysis: This activity involves a more detailed description of the individual tasks including their input and output objects, the source material handled within or delivered by the task, control relations between tasks along with constraints that govern the execution of each task, as well as the roles performing it and so on. Moreover, every task in the process is assessed through its contribution to the core activities of Knowledge Management, i.e. generate, store, distribute and apply knowledge.

Step 4: Business Process Design: This activity involves the modelling of the business process using a graphical tool. The output of this step is a BP model enhanced with KM tasks for the knowledge flow in the BP. Based on the assessment of tasks from the previous step, at least the following knowledge-related process improvements are possible:

- Automate tasks and add automatable tasks: Some knowledge-related tasks, in particular information retrieval (IR) and information gathering tasks, can be fully or partially automated. To this end, an IR application is started and fed with relevant input (e.g., search terms, search context, cp. [Abecker et al. (2000)]), and actively delivers task-specific explicit knowledge [Abecker et al. (2002)]. In order to maintain some process-instance specific task context, the BP control flow may be extended by additional variables, called context variables which transport IR specific information between BP tasks and to the IR algorithms.

- Close knowledge cycles: If a gap in the sequence of knowledge-related tasks is identified, it is filled by adding the corresponding tasks. For example, if somewhere in the process the generation of knowledge has been identified but this knowledge is not stored, a KM task for storing this knowledge can be added in the business process. Heisig (2000) proposes proven best-practice instruments to close knowledge cycles.

- Add KM sub-processes: Some operative BPs can easily be enhanced by interleaving them with (standardized) KM reference processes or process parts. For instance, creation and retrieval of lessons learned or best practices could be integrated into the execution of all knowledge-intensive, difficult decisions in BPs. Reference KM processes are discussed in [Karagiannis and Telesko (2000); Bach et al. (2000)].

Step 5: Ontology Creation: This activity involves the development of a preliminary ontology taking into account the analysis made during the preceding 
steps. An ontology is a formal, explicit specification of the conceptualisation underlying a given domain of application, as shared between a number of (human or software) actors collaborating or communicating in this domain [see Uschold and Gruninger (1996)]. The data collection process of the ontology creation effort is both an iterative and interactive process. The data collection may occur in different modes (interviews with domain experts, direct transcription of data from source documents etc). Regardless of the data collection methods used, each piece of collected data must be traceable back to its source because it is the data that provides objective evidence for the basic ontology structures that are later isolated from this data. Therefore we use four important support documents to facilitate source data traceability: 1) Source Material Index, 2) Source Material Description Form, 3) Term Pool, and 4) Term Description Form. In the Term Pool and Term description form we record the meaningful Terms relevant to the ontology development project effort. It is from these Terms that we construct an initial ("first pass") characterization of the ontology, i.e. identify the three central concepts comprising the ontology:

- Kinds: are an objective category of objects sharing a set of properties;

- Characteristics: are the properties belonging to a Kind;

- Relations: are the sorts of general features that Kinds exhibit jointly rather than individually.

Step 6: Ontology Refinement: This activity involves the refinement and validation of the ontology. During this step, the ontology structures are "instantiated" (tested) with actual data, and the result of the instantiation is compared with the ontology structure. If the comparison produces any mismatch, every such mismatch must be adequately resolved. Refinements to the initial ontology are incorporated to obtain a validated ontology.

So we see Step 4 (Business Process Design) as the central step to improve processes to include Knowledge Management activities. All modelling activities however are based upon the process modelling formalism described below.

\section{Modelling and Enacting Weakly-Structured Processes}

In this section we describe the technical solution that realises the objectives mentioned in previous sections. The proposed solution consists of a tool for modelling knowledge enhanced BPs with associated information needs and for modelling domain knowledge structures and a workflow engine that enacts the modelled processes and interacts with intelligent agents that play the role of the information 
assistant, observing the running process, interpreting the information needs and offering context-sensitive knowledge storage and retrieval.

\subsection{The Business Process Knowledge Modelling Tool}

\section{Technical basis of the modelling tool}

The Business Process Knowledge modelling tool is built upon the DHC CognoVision ${ }^{\circledR}$ tool [Müller and Herterich (2001)] for document and metadata handling, and upon MS VISIO ${ }^{\circledR}$. CognoVision ${ }^{\circledR}$ is a document-based knowledge archive that creates a logical encapsulation of information objects (documents, web pages, etc), manages meta-data and the attributes of these information objects, and allows for structured views and intelligent semantic links among the information objects. CognoVision ${ }^{\circledR}$ is our basis for the development of the process-oriented structured archive, while the integration of $\operatorname{CognoVision} \AA$ with MS VISIO $®$ is used for modelling the BPs. Our integration of CognoVision ${ }^{\circledR}$ and MS VISIO ${ }^{\circledR}$ maps the MS Visio $\AA$ modelling constructs to structuring elements in CognoVision $®$. Shapes in MS Visio ${ }^{\circledR}$ become information objects in CognoVision ${ }^{\circledR}$ and edges connecting shapes become links. The models developed can later be enacted using the workflow engine. For that, all the information needed is stored as attributes of these information objects and links. Information needs for specific tasks are modelled using an agent-description statement which is interpreted by the workflow engine as a call to a specific agent.

\section{The weak-workflow modelling framework and tool}

In order to model knowledge-related tasks and knowledge objects within weaklystructured BPs on a conceptual level, we construct a workflow meta-model that emphasises the coherence between them [Papavassiliou et al. (2002)]. The basic modelling constructs that are provided for the design of BPs include:

- Tasks: A task represent the structured work in the business process that must be done to achieve some objectives. We can distinguish:

Operative Tasks: They are the "normal" operative things required to get the job done.

- Knowledge Management Tasks: KM Tasks are used to describe the work associated with the generation and application of knowledge in the BP. The execution of a KM task may contribute to the successful performance of an operative task.

- Task Interfaces: A Task Interface is a special kind of task used to connect two different models by linking to the start of a more complex BP seen here as a black box. 
- Automatic Tasks: An automatic task describes work that can be done without any user interaction.

- Events: Events are used to trigger the execution of tasks.

- Connectors: They are used for modelling complex flows in the BP.

- Data Objects: They describe variables used in the model to control the flow of the BP when executed by the workflow engine.

- Knowledge Objects: Knowledge Objects represent the explicit knowledge required in a BP. Knowledge objects facilitate and leverage knowledge creation and sharing activities by providing to humans the information they need. They facilitate the knowledge transfer from persons to persons or from information to persons and are used to search, organise and disseminate knowledge content. Knowledge objects serve as input for Tasks and KM Tasks in the BP model, and they are produced as output.

- Roles: Tasks and KM Tasks are assigned to roles during the BP modelling. They describe the entity that can and is allowed to perform the specific task.

- Persons: Persons describe real employees - users of the tool. When the BP model is enacted, persons are playing the roles that have been modelled.

A knowledge-intensive BP is defined in a workflow model. The workflow model consists of tasks and their interdependencies. Each of these tasks can be decomposed into (sub)tasks, which in turn can represent a whole workflow. So far, we discussed the task and organizational perspectives of our workflow meta-model [Papavassiliou et al. (2002)].

A detailed planning of the work to be done in knowledge-intensive business processes is quite difficult to be achieved in advance. To deal with this observation, in our approach under-specified modelling is allowed. The workflow model can include tasks not completely specified in form of a hierarchically ordered set of black boxes. The specification of such tasks can be completed during run-time with more detailed information.

To cope with the control perspective of workflow modelling, we make the following provisions: Tasks are connected with events using control flow elements (sequence, and, or, xor) forming Event-driven Process chains (EPCs). EPCs are extended by links to other relevant entities. In this way, tasks can be connected to input and output data to model the data flow in the process, and to knowledge objects to model the information flow. The control flow of the business process is modelled using sequences, splitters and joiners. With the sequence flow element, it is possible to link two tasks sequentially. More interesting are the split-join constructions that allow a path in the process to split into multiple parallel branches. It can be specified that such parallel branches all must be executed at the same time (and-split), or that only one (xor-split) or some (or-split) of these branches have to be executed. 


\section{Knowledge enhanced business processes}

In order to support in an integrated manner the modelling of those activities in the BP that are associated with the creation and application of knowledge, we extend the EPCs with additional tasks, the KM tasks. The usage of these tasks has already been explained above in the "BP Design" step. These KM tasks, together with their control flow and the context variables to control their specific behaviour are one major part of what we call the knowledge perspective, in addition to the conventional workflow modelling perspectives. The other important part of this perspective are Ontologies.

According to the Business Knowledge Method, the three central concepts that comprise the constructed ontology are Kinds, Characteristics and Relations. A mapping of these concepts to the specific elements of the CognoVision ${ }^{\circledR}$ document management [Müller \& Herterich (2001)] tool looks as follows:

- $\quad$ Kinds are mapped to structure units

- $\quad$ Characteristics are mapped to definable attributes

- $\quad$ Relations are mapped to links

Therefore, in order to implement the constructed ontology in the tool, one should take into account the previous mapping, and use structure units to hierarchically structure the information, definable attributes to identify the properties of each structure unit, and finally links to represent not only the relations holding between structure units, but also to link to respective information/knowledge sources.

Consequently, one starts by the CognoVision ${ }^{\circledR}$ Administrator where the different types of structure units, definable attributes and links have to be defined before they can be used to implement the ontology. The next step is to implement the ontology in the CognoVision ${ }^{\circledR}$ client. Therefore, one uses the previously defined structure units with their assigned definable attributes to hierarchically structure the information, and links to represent the relations between structure units. Links are also used to link structure units to the respective information / knowledge sources (e.g. MS Word documents, adobe acrobat documents, html files etc.), provided that all such information sources are first imported into the system using the import functionality of the CognoVision ${ }^{\circledR}$ client. Thus, the process-oriented knowledge archive for the selected business process is created.

\subsection{Enacting the Weakly-Structured Business Process}

Up to now we have implemented a simple, strongly-structured workflow engine closely coupled to CognoVision®. The weak-workflow functionalities can also be demonstrated with simple KM services, but not yet seamlessly integrated with CognoVision®. Nevertheless, we will focus here on weak-workflow enactment, 
since this scientifically much more interesting, and the missing technical integration is only an implementation issue, not a principal problem. This weak-workflow enactment has been designed as an agent-based integrated weak-workflow / Organisational Memory approach (see [Abecker et al. (2003)] implemented using the FIPA-compliant agent platform JADE [Bellifemine et al. (2001)].

The workflow engine interprets the process logic represented in the BP Model, goes through the modelled tasks and assigns each task to specific users according to the associations that have been modelled in the responsibility diagram. Whenever an information need is associated with a task as already described during modelling time, the workflow engine invokes an intelligent agent in the background. Given the corresponding context from the workflow task (modelled with data objects), the agent accomplishes an ontology-based information search in the process-oriented structured archive to satisfy the user's information need by presenting the relevant information.

The different functionalities of our approach are supported by various agents. Figure 2 gives an idea about the several agents with typical communicative acts.

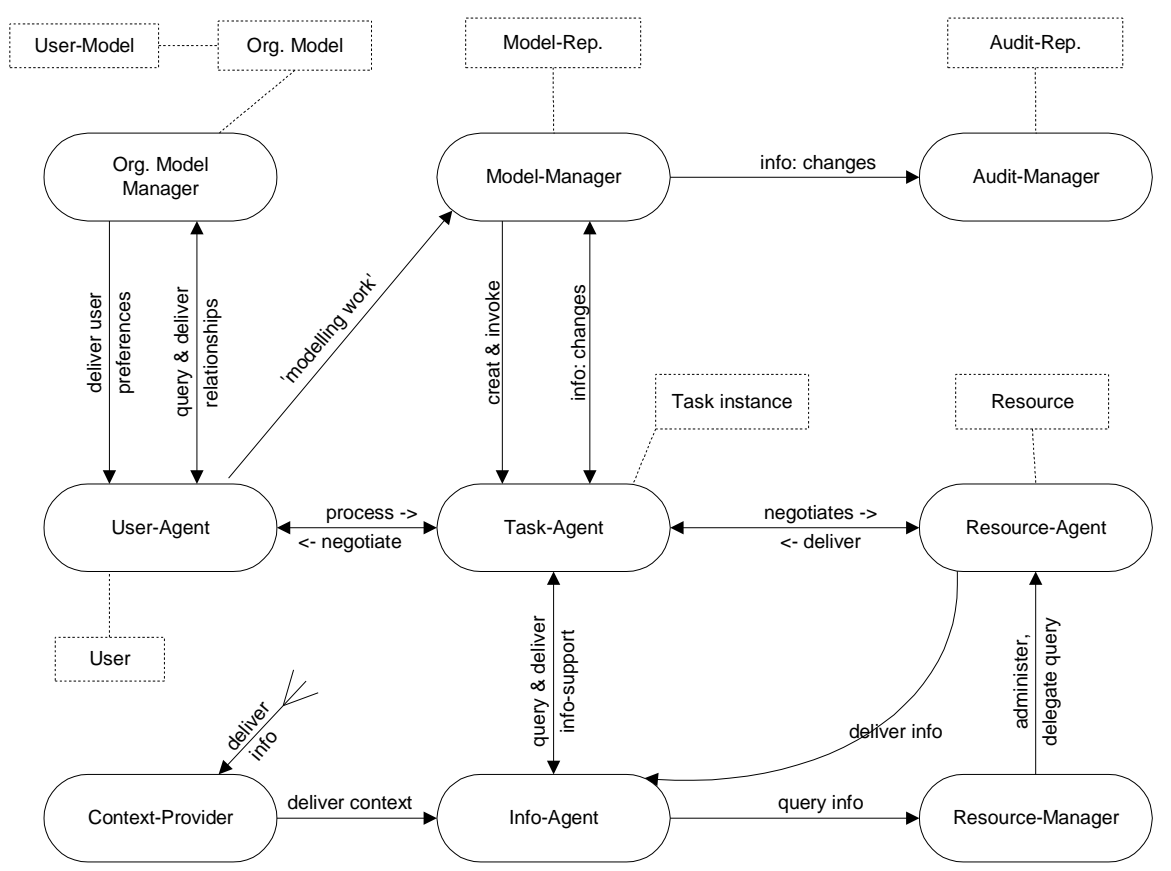

Figure 2: Communication between agents

The ContextProvider uses all accessible information sources in the environment, including their information offers, heuristics for context delivery and the context model in order to offer useful and tailored context information. 
The InfoAgent uses the context information offered by the context agent, the information/knowledge resources and the domain ontology in order to provide the right knowledge at the right time, contextually embedded.

The ResourceManager handles the resource agents. Its main services include requests for resources with given features, direct search for a specific resource agent, requests for information with specific features and negotiation with several resource agents about information delivery.

The ResourceAgent is used for information delivery for specific queries and for negotiation about this information delivery and about resource usage.

Finally, the UserAgent handles read/write access to roles for the end users and for handling their worklist. It cooperates with the task agent for the task execution.

The OrganisationalModelManager is responsible for the Organisational Model. Its main services include answering questions about the organisational structure.

The ModelManager handles the repository of the workflow models. The goal of that agent is to associate a task agent to every task that is not fully subsumed by its subtasks. It is responsible for managing read/write access to models, instances, concepts and embedded data, for searching for models, instances and concepts referring to ontological criteria and for copying /instantiating a model. The competencies of that agent include queries to domain ontologies, creations and start of a task agent for a task instance and informing the audit manager about new or changed modelling decisions.

The AuditManager handles the audit repository. It is used for performing queries about modelling history (models, modelling changes, old model versions, changes actions within a task instance model) and for storing a new model/instance.

TaskAgents are responsible for the successful completion of tasks. Their main actions include queries to domain ontologies, negotiations with user agents and resource agents about task execution and knowledge provision and modifications to the model of the respective task instance.

\section{Public Administration as an Application Area}

The following four points outline the relationship of the main elements of our solution to public administration processes:

The idea of weak workflow structures is required, since normally, legal regulations only provide a process skeleton while specific knowledge-intensive tasks [Buckingham (1998)] are below the granularity normally modelled [Dellen et al. (1997)] - this is what Lenk and Traunmüller see as a specific characteristic of egovernment processes: "They are partly ... structured by legal rules which how- 
ever, often demand interpretation ..." [Lenk and Traunmüller (2000)]; or because during long-living administrative process instances rules may change [Dellen et al. (1997)]; or because specific exceptions may occur once for the first time.

The idea of active knowledge delivery is especially useful since not all employees dealing with a given topic have the same education and expertise, and decisions along given binding regulations must be ensured. Active hints to other's decisions are useful to guarantee equal decisions under equal conditions. Further they support the dissemination of new knowledge, for instance in the case of changed laws, etc. In the case of "normal citizens" or not deeply specialized operators interacting with a system, the active delivery fosters democracy since it allows to profit from and be part of complex processes without having all required background knowledge in advance. Moreover, it supports legal validity and transparency even in such cases as described in Lenk and Traunmüller's "innovative ways of service delivery" [Lenk and Traunmüller (2000)].

In governmental processes ontology-based Organisational Memory systems are especially important: many existing sources of knowledge, laws, comments to laws, specific regulations, old similar cases, available case-specific documents and information etc, are prevalent at different places and in different forms and representations, at several degrees of formality, and related by manifold links. In order to make informed, transparent, and accountable decisions, consistent with the past, compliant with the law, and coherent with similar decisions in other places, all this information should be placed into a coherent framework. Having this framework on a formal basis allows sophisticated assessment of relevance in information retrieval (e.g., by case-based reasoning methods).

In the area of public administration, our Business Process Knowledge method is definitely required because, without a deep understanding and sound approach to $\mathrm{KM}$ activities, the ever growing complexity of bureaucracy in a networked Europe will soon make impossible informed administrative decisions which properly take into account all relevant, actual information. Further, the increasing speed of change in administrative regulations and processes requires adequate organisational processes to keep pace with the changing world. This is even more the case since (especially in critical decisions) the citizens have access to the same information sources as the officials such that sub-optimal decisions are not longer accepted. A methodologically sound approach is also necessary because the public sector is so big that one needs clearly defined procedures in order to come to comparable (and, technically, interoperable) results.

\section{The IKA case}

Below we present an application of our approach in a specific knowledge-intensive business process. We tested our approach in an organisation from the social security sector: the Greek Social Security Institute (IKA), which is the largest insurance institution in Greece. Having as its primary purpose the protection of the in- 
sured persons, IKA offers an extensive range of services to them, like insurance, benefits, pensions and interstate social security. Currently, IKA provides health care to 5.500 .000 insured persons including the members of their family and pays out pensions to 1.000 .000 pensioners approximately.

\section{Description of the business process}

The process that was examined and modelled with our tool is the granting of full old age pension. The significance of the pension process lies in the large number of beneficiaries that currently amounts to 1.000 .000 persons and increases at an annual rate of $10 \%$. In addition, the pension granting process requires a deep knowledge of the relevant legislation; first for making the decision whether the insured person is entitled to receive a pension; and second for calculating the amount of pension.

It is quite common that for one specific case more than one legal regulation may be relevant, and it is a matter of knowledge and experience to identify all these regulations and then choose the most appropriate one. If it is the case that the insured member can establish a pension right under more than one regulation, the different pension amounts are calculated, and the highest one is chosen. In addition, the pension granting process - as part of a normal administrative workflow - contains some central, knowledge and document intensive steps for coming to a decision whether the insured person is entitled to receive a pension or not, and to calculate the correct pension amount. These steps must be legally checkable, they are often done with uncertainty, based on the experience of the relevant regulations the employees have and they are vital for the correct result of the process.

The process begins with the submission of the application form by the insured person and the collection of all the supplementary documentation, which constitutes the retirement folder. The retirement folder is submitted by the insured person to any of IKA's branches and then it is forwarded to the one being responsible for acting upon it. The pension folder is being checked at the department of pensions or the department of payments. If it is not complete, a communication between the department of pensions or the department of payments and the insured member or other departments or even other branches takes place in order to receive the documents that are required for the establishment of the pension right. The insured person is entitled to pension when he/she fulfils the prerequisite conditions (e.g., minimum number of working days and age) for the specific type of pension and category to which he/she belongs. The decision regarding the entitlement to a pension is made on the basis of the employment and personal data of the insured person. This decision is based also on the current legal regulations, which are differentiated according to the pension type, the category of the insured person and other factors. Having established that the minimum prerequisite conditions are met, a decision of approval is issued, which mentions all the information related to the granting and the calculation of the pension. If the insured person is not entitled to a pension, a decision of rejection is issued. 
After application of the Business Process Knowledge Method, the model of IKA's "Granting of full old age pension" business process was developed and enhanced with Knowledge Management tasks. Figure 3 depicts a part of the model as it is presented to the user. The business process model is presented as HTML pages where the shapes are anchors for links in the knowledge archive. This means that the models can be used for navigating a knowledge network. By clicking on an object of the model the user is presented with attributes for the specific object. The respective ontology was implemented in the DHC CognoVision ${ }^{\circledR}$ component of the Business Process Knowledge Modelling Tool (see Figure 4).

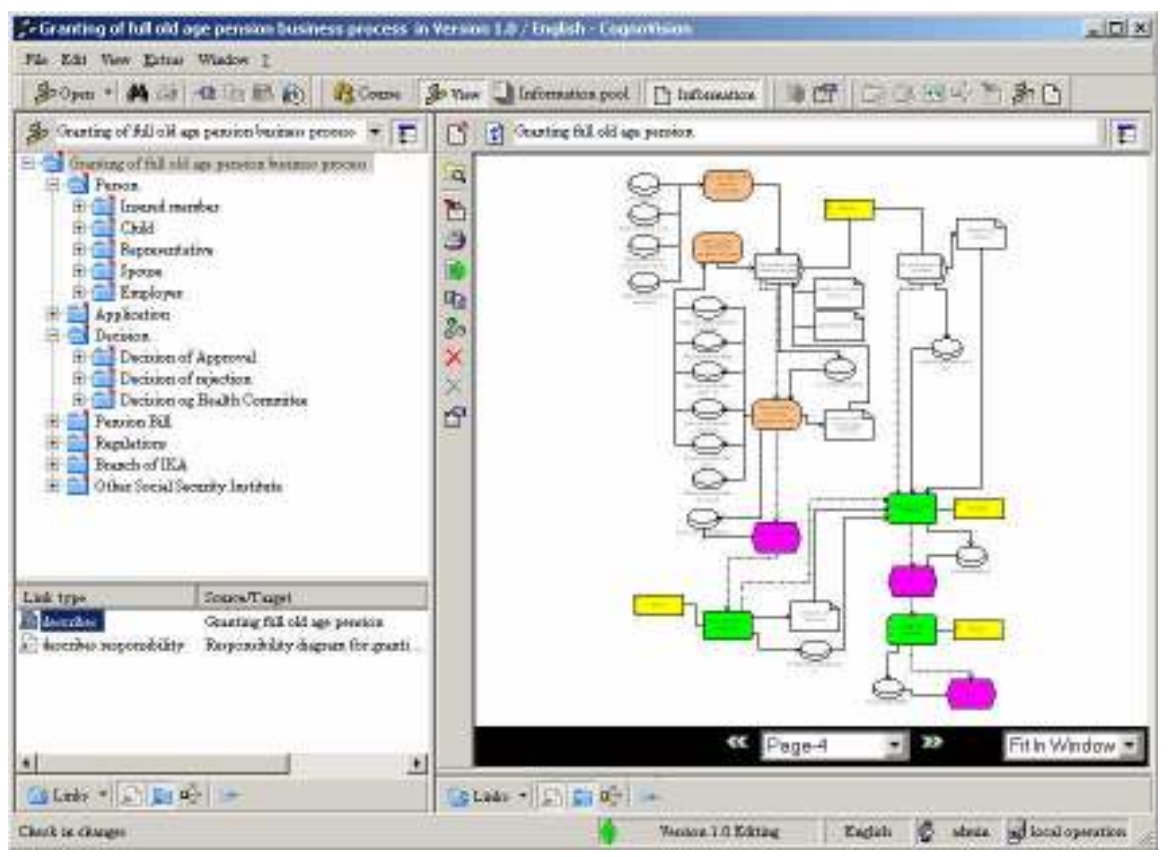

Figure 3: Part of IKA's business process model 


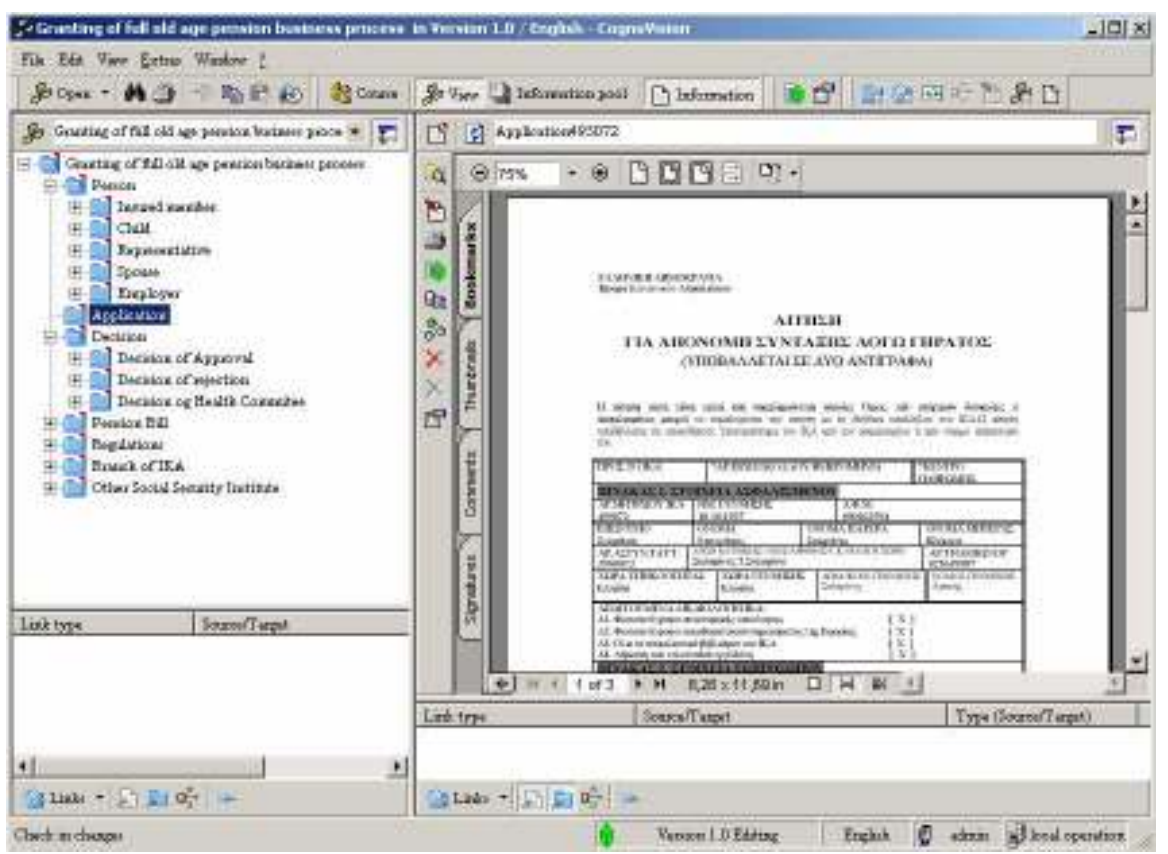

Figure 4: The process-oriented knowledge archive of IKA's "granting of full old age

Our first usage experiences with the DECOR prototypes were extremely positive. However it turned out that detailed, quantitative measurements for the positive effects of BPOKM solutions are very difficult. Just to give a flavour of achievable results, we shortly describe some IKA experiments: Following the initial test and after ensuring the proper operation of the prototype in terms of workflow execution of the BP, a training workshop with the IKA personnel was organised. The demonstration of the system involved first processing with the system two past cases by ICCS / PLANET-EY for demonstrating the system functionality. Then, three other past cases were processed by the IKA personnel. The next step was the operation of the system by IKA personnel with 15 past cases (again different from all the previously entered in the system) in order to fill in the archive and create an initial knowledge base with similar cases (Lessons Learned). The cases were carefully selected in order to be representative and contained at least one occupation category (e.g. construction workers, syndicalists), both sexes and spanned across different age ranges. Finally the system was tested again by the IKA personnel with 15 new cases. These cases were applications of insured members recently submitted to IKA for which no decision had been issued yedt. During this phase indicative time measurements were taken in order to derive an initial assessment of the speed in executing the business process with the aid of the tool. Roughly, the following quantitative measurements for the effect of the tool were observed: 


\begin{tabular}{|l|l|l|}
\hline Criteria & $\begin{array}{l}\text { Reference } \\
\text { measurement }\end{array}$ & With DECOR \\
\hline $\begin{array}{l}\text { Number of decisions issues per day (in case all } \\
\text { the respective documentation is available to the } \\
\text { person examining the application in order to } \\
\text { issue a decision) }\end{array}$ & 2,4 & 4 \\
\hline $\begin{array}{l}\text { Number of decisions issued per week against } \\
\text { the number of submitted applications per week }\end{array}$ & $21,86 \%$ & $43 \%$ \\
\hline Percentage of appeals to IKA's decision & $10 \%$ & $9 \%$ (estimated) \\
\hline
\end{tabular}

Table 1: Qualitative results of tool usage

Some more information about the test runs can be found in [Abecker et al. (2003b)]. More experiments about the effect of using weak-workflow tools with coupled context-sensitive information support have been reported by [Elst et al. (2003)].

\section{Related Work, Conclusions and Further Research}

The links between BPs and KM have extensively been discussed by Heisig (2000) who presents an approach to analyse BPs from a KM perspective, and tries to integrate KM activities into daily business. Starting from the selection of the business area and process, every task which is considered to be a knowledge processing task is assessed through its function and contribution to the core activities of KM (i.e. generate, store, distribute, apply knowledge) resulting in a knowledge activity profile which shows the level of support provided by the operational task towards the core process of KM. The BP is improved by closing identified gaps and by sequencing the KM core tasks.

The model-based KM approach proposed by Allweyer (1998) adds a new perspective to the modelling of existing BPs, especially of knowledge-intensive processes. KM activities are considered as an integral part of BPs. The four level BPM architecture is adopted for $\mathrm{KM}$, resulting in the knowledge process redesign method. The approach aims at the description of required and used knowledge as well as generated and documented knowledge. The approach supports structuring of knowledge into categories and the construction of a knowledge map to locate who knows what inside the organisation. Easy-to-understand pictograms are proposed to help users describe the use of documented and tacit knowledge within their BPs. The approach does not make explicit how to integrate the KM activities into 
BPs and does not provide criteria to analyse and improve the knowledge processing within the BP.

The idea of interrelating Knowledge Management Processes (KMPs) and Business Processes is also a main topic of the EU project PROMOTE [Karagiannis and Telesko (2000)] which has similar analysis goals and methods as DECOR. Their method consists of five steps: Strategic Decisions - the Awareness phase, KMP Analysis, KMP and OM Modelling, Specification and Implementation, Evaluation and Continuous Optimisation. In PROMOTE, a Knowledge Builder is developed allowing users to model KMPs describing the knowledge flow in the business process. BP models are used to define when to access the OM, and KMP processes are used to define how to access the OM. However, KM activities and BP tasks are not explicitly integrated in the modelling phase.

Ontologies have shown to be the right answer to structuring and modelling problems by providing a formal conceptualisation of a particular domain that is shared by a group of people in an organisation. A methodology for ontology-based KM can be found in [Staab et al. (2001)]. The methodology extends and improves the CommonKADS methodology by introducing specific guidelines for developing and maintaining the specific ontology. The most interesting part in contrast to other methodologies for ontology development is that this approach focuses on the application-driven development of ontologies.

In this paper we presented an innovative approach that supports knowledge-intensive BP by proactively offering to the users dealing with specific tasks contextsensitive knowledge from a knowledge archive. The proposed solution includes a powerful modelling tool that supports in an integrated manner the modelling of weakly-structured BP and domain knowledge structures, and an engine that enacts such processes. Our solution is accompanied by a Business Process Knowledge method that offers guidance for introducing such a solution into an organisation.

Next steps in our work include the finalisation of the weak-workflow enactment and the interfaces between the workflow engine and the intelligent agents that perform the context-sensitive knowledge retrieval and storage. Furthermore, we work towards providing electronic support for all the steps of our method. This means that we want to equip users with electronic forms that facilitate the gathering of all the information that are presently captured by hand, and also provide a graphical representation for drawing the domain ontology by adopting some of the symbols of the IDEF5 Schematic Language.

Acknowledgment: Part of this research work has been carried out in the context of the DECOR project, which was partly funded by the European Commission, IST Programme KA Action II (New Methods of Work and Electronic Commerce), under Grant IST-199913002. During the DECOR project runtime, the last author of this paper was with the German Research Center for Artificial Intelligence (DFKI) Kaiserslautern, and acted as the project coordinator. Other parts of the work presented is based on the results of the project FRODO: A Framework for Distributed Organisational Memories (also running at DFKI, 
funded by the German Federal Ministry for Education and Research, bmb+f, under Grant 01 1W 901).

\section{References}

Abecker, A., and Mentzas, G. (2001), Active Knowledge Delivery In Semi-Structured Administrative Processes. M. Wimmer (ed) Knowledge Management in Electronic Government, KMGov-2001, pp. 47-57,

Abecker, A., Bernardi, A., Hinkelmann, K., Kühn, O., and Sintek, M. (2000), ContextAware, Proactive Delivery of Task-Specific Knowledge: The KnowMore Project. Int. Journal on Information Systems Frontiers (ISF) 2(3/4):139-162, Special Issue on Knowledge Management and Organizational Memory, Kluwer.

Abecker, A., Bernardi, A., Sintek, M., and Hinkelmann, K. (2002), Enterprise Information Infrastructures for Active, Context-Sensitive Knowledge Delivery. In: S. Barnes (ed.) Knowledge Management Systems: Theory and Practice. International Thomson Business Press.

Abecker, A., Bernardi, A., Elst, L. van (2003), Agent Technology for Distributed Organizational Memories. In: ICEIS-2003 - 5th Int. Conference on Enterprise Information Systems, Angers, France, April 2003.

Abecker, A., Papavassiliou, G., Ntioudis, S., Mentzas, G., and Müller' S. (2003b), Methods and Tools for Business-Process Oriented Knowledge Management: Experiences from Case Studies. In: ICE 2003 - 9th International Conference of Concurrent Enterprising, Espoo, Finland, 16-18 June 2003.

Allweyer, Th. (1998), Modellbasiertes Wissensmanagement. In: Information Management, $1,37-45$.

Bach, V., Oesterle, H., and Vogler, P. (2000), Business Knowledge Management in der Praxis, Berlin Heidelberg: Springer Verlag. In German.

Bellifemine, F., Poggi, A., and Rimassa, G. (2001), Developing multi agent systems with a FIPA-compliant agent framework. Software - Practice and Experience, 31:103-128.

Buckingham Shum, S. (1998), Negotiating the Construction of Organisational Memories, in U.M. Borghoff, and R. Pareschi, R., (eds.), Information Technology for Knowledge Management. Berlin, Heidelberg, New York: Springer-Verlag, pp. 55-78.

Dellen, B., Pews, G. and Maurer, F. (1997), Knowledge Based Techniques to Increase the Flexibility of Workflow Management. Data \& Knowledge Engineering Journal, NorthHolland.

Elst, L. van, Aschoff, F.-R., Bernardi, A., Maus, H., Schwarz, S. (2003). Weakly-structured Workflows for Knowledge-intensive Tasks: An Experimental Evaluation. Accepted for: Workshop Knowledge Management for Distributed Agile Processes: Models, Techniques, and Infrastructure (KMDAP2003) at WETICE-03. 
Heisig, P. (2000). Process Modelling for Knowledge Management. In EKAW Workshop on Common Approaches on Knowledge Management, 12th International Conference on Knowledge Engineering and Knowledge Management, Juan-les-Pins, French Riviera.

IDEF5 Ontology Description Capture Overview (2000), available at http://www.idef.com .

Karagiannis, D., and Telesko, R. (2000), The EU-Project PROMOTE: A Process-Oriented Approach for Knowledge Management. In Reimer, U. (ed.) PAKM 2000, Third Int. Conf. on Practical Aspects of Knowledge Management.

Klein, M., Dellarocas, Ch., and Bernstein A. (2000), Introduction to the special issue on adaptive workflow systems, in Computer Supported Cooperative Work, Vol. 9, pp. 265-267, Kluwer.

Lenk, K. and Traunmüller, R. (2000), Perspectives on Electronic Government, presented at the IFIP WG 8.5 Conference on „Advances in Electronic Government“, Zaragoza, 10$11 / 2 / 2000$.

Müller, S. and Herterich, R. (2001), Prozessorientiertes Wissensmanagement mit CognoVision, presented at the Workshop „Geschäftsprozeßorientiertes Wissensmanagement" at the WM-2001 Conference, Baden-Baden, Germany.

Papavassiliou G., G. Mentzas and A. Abecker (2002), Integrating knowledge modelling in business process management. In $10^{\text {th }}$ European Conference on Information Systems (ECIS 2002), Gdansk, Poland.

Reimer, U., Margelisch, A., and Staudt, M. (2000), EULE: A Knowledge-Based System to Support Business Processes. Knowledge-based Systems Journal, Vol. 13 No. 3, Elsevier.

Schreiber, G., Akkermans, H., Anjeiwerden, A., de Hoog, R., Shadbolt, N., van de Velde, W. and Wielinga B. (1999), Knowledge Engineering and Management: The CommonKADS Methodology. MIT Press.

Staab, St, Schnurr, H.P., Studer, R., and Sure, Y. (2001), Knowledge Processes and Ontologies, In IEEE Intelligent Systems, Special Issue on Knowledge Management.

Staab, St., and Schnurr, H.P. (2000), Smart Task Support through Proactive Access to Organizational Memory. Knowledge-based Systems, Vol. 13 No. 5: 251-260. Elsevier.

Uschold, M., and Gruninger, M. (1996), Ontologies: Principles, methods and applications. In Knowledge Sharing and Review, 11(2), June 1996.

Van Kaathoven, R., Jeusfeld, M., Staudt, M., and Reimer, U. (1999), Organisational Memory Supported Workflow Management, Electronic Business Engineering, Physica Verlag, pp. 543-563.

Wiig, K.M. (1995), Knowledge Management Methods. Practical Approaches to Managing Knowledge. Vol. 3. Schema Press, Arlington.

Yu, B., and Wright, T.D. (1997), Software tools supporting business process analysis and modeling. Business Process Management Journal, Vol.3 No.2, 1997, pp.133-150, MCB University Press. 